\title{
Archiv für Reformationsgeschichte
}

Internationale Zeitschrift

zur Erforschung der Reformation und ihrer Weltwirkungen, herausgegeben im Auftrag des Vereins für Reformationsgeschichte und der Society for Reformation Research

\section{Archive for Reformation History}

An international journal

concerned with the history of the Reformation and its significance in world affairs, published under the auspices of the Verein für Reformationsgeschichte and the Society

for Reformation Research

\section{Herausgeber - Board of Editors}

Jodi Bilinkoff, Greensboro/North Carolina - Michael Driedger, St. Catharines/ Ontario - Renate Dürr, Tübingen - Birgit Emich, Erlangen-Nürnberg Mark Greengrass, Sheffield - Brad S. Gregory, Notre Dame/ Indiana - Scott Hendrix, Princeton/New Jersey - Mack P. Holt, Fairfax/Virginia Susan C. Karant-Nunn, Tucson/Arizona - Thomas Kaufmann, Göttingen - Yves Krumenacker, Lyon - Ute Lotz-Heumann, Tucson/Arizona - Janusz Małtek, Toruń-Silvana Seidel Menchi, Pisa - Carla Rahn Phillips, Minneapolis/Minnesota-HeinzSchilling, Berlin-Anne Jacobson Schutte, Charlottesville/Virginia - Ethan H. Shagan, Berkeley/ California - Christoph Strohm, Heidelberg - James D. Tracy, Minneapolis/Minnesota - Alexandra Walsham, Cambridge - Randall C. Zachman, Notre Dame/Indiana

\section{Redaktion - Managing Editors}

Ute Lotz-Heumann - Brad S. Gregory - Randall C. Zachman

\section{Auflage}

Copyright (C) 2016 by Gütersloher Verlagshaus, Gütersloh, in der Verlagsgruppe Random House $\mathrm{GmbH}$,

Neumarkter Straße 28, 81673 München

Druck und Einband: Hubert \& Co., Göttingen · Printed in Germany

Umschlaggestaltung: Peter Steiner, Stuttgart

ISBN 978-3-579-08470-1

ISSN 0003-9381

www.gtvh.de 


\title{
Archive for Reformation History
}

An international journal

concerned with the history of the Reformation and its significance in world affairs, published under the auspices of the Verein für Reformationsgeschichte and the Society for Reformation Research

\section{Board of Editors}

Jodi Bilinkoff, Greensboro/North Carolina - Michael Driedger, St. Catharines/Ontario - Renate Dürr, Tübingen - Birgit Emich, Erlangen-Nürnberg - Mark Greengrass, Sheffield - Brad S. Gregory, Notre Dame/Indiana - Scott Hendrix, Princeton/New Jersey - Mack P. Holt, Fairfax/Virginia - Susan C. Karant-Nunn, Tucson/ Arizona - Thomas Kaufmann, Göttingen - Yves Krumenacker, Lyon - Ute LotzHeumann, Tucson/Arizona - Janusz Małłek, Torun - Silvana Seidel Menchi, Pisa Carla Rahn Phillips, Minneapolis/Minnesota - Heinz Schilling, Berlin - Anne Jacobson Schutte, Charlottesville/Virginia - Ethan H. Shagan, Berkeley/California - Christoph Strohm, Heidelberg - James D. Tracy, Minneapolis/Minnesota -

Alexandra Walsham, Cambridge - Randall C. Zachman, Notre Dame/Indiana

\author{
North American Managing Editors \\ Brad S. Gregory - Randall C. Zachman
}

European Managing Editor

Ute Lotz-Heumann

Vol. $107 \cdot 2016$ 


\title{
Archiv für Reformationsgeschichte
}

\author{
Internationale Zeitschrift \\ zur Erforschung der Reformation und ihrer Weltwirkungen, \\ herausgegeben im Auftrag des Vereins für Reformationsgeschichte und \\ der Society for Reformation Research
}

\section{Herausgeber}

Jodi Bilinkoff, Greensboro/North Carolina - Michael Driedger, St. Catharines/Ontario - Renate Dürr, Tübingen - Birgit Emich, Erlangen-Nürnberg - Mark Greengrass, Sheffield - Brad S. Gregory, Notre Dame/Indiana - Scott Hendrix, Princeton/New Jersey - Mack P. Holt, Fairfax/Virginia - Susan C. Karant-Nunn, Tucson/ Arizona - Thomas Kaufmann, Göttingen - Yves Krumenacker, Lyon - Ute LotzHeumann, Tucson/Arizona - Janusz Małłek, Toruń - Silvana Seidel Menchi, Pisa Carla Rahn Phillips, Minneapolis/Minnesota - Heinz Schilling, Berlin - Anne Jacobson Schutte, Charlottesville/Virginia - Ethan H. Shagan, Berkeley/California - Christoph Strohm, Heidelberg - James D. Tracy, Minneapolis/Minnesota -

Alexandra Walsham, Cambridge - Randall C. Zachman, Notre Dame/Indiana

Europäische Redaktion

Ute Lotz-Heumann

Nordamerikanische Redaktion

Brad S. Gregory - Randall C. Zachman

Vol. $107 \cdot 2016$ 


\author{
Mitarbeiter der Redaktion - Editorial Assistant \\ Dr. Christian Jaser \\ Humboldt-Universität Berlin \\ Institut für Geschichtswissenschaften
}

Das Archiv für Reformationsgeschichte erscheint jährlich in einem normalerweise 320 Seiten umfassenden Aufsatzband und einem ca. 192 Seiten umfassenden Literaturbericht (Beiheft).

Manuskripte aus Europa werden per E-Mail erbeten an Prof. Dr. Ute Lotz-Heumann, ulotzh@email.arizona.edu (Postadresse: Division for Late Medieval and Reformation Studies, University of Arizona, Douglass 307, PO Box 210028, Tucson, AZ 85721-0028, USA). Manuskripte aus Nordamerika werden per E-Mail erbeten an Prof. Dr. Randall C. Zachman, rzachman@nd.edu (Postadresse: 130 Malloy Hall, University of Notre Dame, Notre Dame, IN 46556, USA). Es werden nur Original-Beiträge aufgenommen. Es wird empfohlen, rechtzeitig vor Abschluss des Manuskripts bei der jeweiligen Redaktion Merkblätter zur formalen Gestaltung der Beiträge anzufordern. Besprechungsexemplare aus Europa und Nordamerika werden erbeten an Prof. Dr. Markus Wriedt, Goethe-Universität Frankfurt am Main, Fachbereich Evangelische Theologie, Professur für Kirchengeschichte, Norbert-Wollheim-Platz 1, D-60323 Frankfurt am Main, E-Mail: m.wriedt@em.uni-frankfurt.de.

The Archive for Reformation History appears annually in one volume consisting usually of 320 pages containing the essays and a supplement volume consisting of 192 pages containing the literature review.

Manuscripts and communications concerning editorial matters originating in North America should be sent by e-mail to Professor Randall C. Zachman, rzachman@nd.edu. His postal address is 130 Malloy Hall, University of Notre Dame, Notre Dame, IN 46556, USA. Manuscripts and communications originating in Europe should be sent by e-mail to Professor Ute Lotz-Heumann, ulotzh@email.arizona.edu (postal address: Division for Late Medieval and Reformation Studies, University of Arizona, Douglass 307, PO Box 210028, Tucson, AZ 85721-0028, USA). Only original manuscripts are accepted. Authors' guides for the preparation of manuscripts are available from either the North American or the European managing editors. Books, journal articles, and offprints for review originating in North America or Europe should be sent to Professor Markus Wriedt, Goethe-Universität Frankfurt am Main, Fachbereich Evangelische Theologie, Professur für Kirchengeschichte, Norbert-Wollheim-Platz 1, 60323 Frankfurt am Main, Germany, m.wriedt@em.uni-frankfurt.de.

Auch als Online-Ausgabe erhältlich: www.degruyter.com/view/j/arg

ISBN 978-3-579-08470-1

Print-Ausgabe: ISSN 0003-9381

Online-Ausgabe: ISSN 2198-0489

Copyright (C 2016 by Gütersloher Verlagshaus, Gütersloh, in der Verlagsgruppe Random House $\mathrm{GmbH}$,

Neumarkter Straße 28, 81673 München

Die Zeitschrift und alle in ihr enthaltenen einzelnen Beiträge und Abbildungen sind urheberrechtlich geschützt. Jede Verwertung außerhalb der engen Grenzen des Urheberrechtsgesetzes ist ohne Zustimmung des Verlages unzulässig und strafbar. Das gilt insbesondere für Vervielfältigungen, Übersetzungen, Mikroverfilmungen und die Einspeicherung und Verarbeitung in elektronischen Systemen.

Satz: SatzWeise GmbH, Trier

Druck und Einband: Hubert \& Co., Göttingen

Printed in Germany

www.gtvh.de 